\title{
A Next Generation Integrated Environment for Collaborative Work Across Internets
}

\author{
Final Report - Grant No: DE-FC03-99ER25419
}

\author{
Principal Investigator: Harvey B. Newman \\ newman@hep.caltech.edu
}

$\begin{array}{lll}\text { Co-Investigators: } & \begin{array}{l}\text { Philippe Galvez } \\ \text { Gregory Denis }\end{array} & \begin{array}{l}\text { (Caltech) } \\ \text { (Caltech) }\end{array} \\ & \text { James F. Leighton } & \text { (ESnet) } \\ & \text { Stan Kluz } & \text { (ESnet) }\end{array}$

\author{
California Institute of Technology \\ 1200 East California Blvd. \\ Pasadena, CA 91125
}

(626)-395-6656; Fax (626)-795-3951

Collaborators: $\quad$ CERN, Internet2/UCAID

\section{Introduction; Overview}

\begin{abstract}
We are now well-advanced in our development, prototyping and deployment of a high performance next generation Integrated Environment for Collaborative Work. The system, aimed at using the capability of ESnet and Internet2 for rapid data exchange, is based on the Virtual Room Videoconferencing System (VRVS) developed by Caltech. The VRVS system has been chosen by the Internet2 Digital Video (I2-DV) Initiative as a preferred foundation for the development of advanced video, audio and multimedia collaborative applications by the Internet2 community. Today, the system supports high-end, broadcast-quality interactivity, while enabling a wide variety of clients (Mbone, H.323) to participate in the same conference by running different standard protocols in different contexts with different bandwidth connection limitations, has a fully Webintegrated user interface, developers and administrative APIs, a widely scalable video network topology based on both multicast domains and unicast tunnels, and demonstrated multiplatform support. This has led to its rapidly expanding production use for national and international scientific collaborations in more than 60 countries.
\end{abstract}

We are also in the process of creating a "testbed video network" and developing the necessary middleware to support a set of new and essential requirements for rapid data exchange, and a high level of interactivity in large-scale scientific collaborations. These include a set of tunable, scalable differentiated network services adapted to each of the data streams associated with a large number of collaborative sessions, policy-based and network state-based resource scheduling, authentication, and optional encryption to maintain confidentiality of inter-personal communications. High performance testbed video networks will be established in ESnet and Internet2 to test and tune the implementation, using a few target application-sets.

\section{Tasks Description and Progress}

\section{$\underline{2.1 \text { Introduction; Overview }}$}

The Caltech "Virtual Rooms Videoconferencing System" (VRVS) ${ }^{1}$ has been developed since 1995 to provide a low cost, bandwidth-efficient, extensible means for videoconferencing and remote collaboration over networks within the High Energy and Nuclear Physics communities. Since it went into production service in early 1997, VRVS has become a standard part of the toolset used daily by a large sector of HEP, and it is used increasingly for other DoE-supported programs. It has also attracted substantial interest in diverse fields of science and engineering outside the HEP.

The VRVS Web-based system is now deployed on more than 10,500 registered hosts running the VRVS software

\footnotetext{
${ }^{1}$ See http://www.vrvs.org
} 
in more than 60 countries. There are currently 42 "reflectors" that create the interconnections and manage the traffic flow, in the Americas, Europe and Asia. New reflectors recently have been installed in Brazil, Romania, Portugal, and 5 new reflectors have been deployed over the Internet2 Backbone.

VRVS is based on the Virtual Rooms concept. Participants at different geographical locations set up a videoconference (audio, video and shared applications) in a unique virtual area, simply by pointing and clicking on the icons representing the desired services. This concept is implemented using two technologies. On one hand, a series of IP Servers/Reflectors connect users to a Virtual Room (setting up a series of interconnected IP tunnels), forming a private video-group. On the other hand, the Web user-interface provides worldwide secure access on demand (for registered users), to each Virtual Room.

Booking a virtual room is performed by the user through the web-oriented scheduler, in the same way he would book a local conference room, but independently of his physical location. One can change or cancel a booking in a familiar way. If all the virtual rooms are already booked, it means that the (pre-set) maximum number of parallel videoconferences has already been reached.

The VRVS system is managed by the Caltech CMS group. During the year 2001, following the deployment of the new version (V2.5) in May 2001, we had an increase by approximately a factor of two in the number of users registered in the system. The number of multipoint collaborative sessions (national and international) using VRVS increased by $110 \%$ as well. This high adoption rate confirmed the need within the Research and Academic community for performing and easy-to-use collaborative tools. Integration with the AccessGrid technology ${ }^{2}$ has been successful, and the VRVS/AG gateway (VAG), where one can join any Access Grid Virtual Venue from a desktop or laptop, is heavily used by the community.

A new version of VRVS (V3.0) is about to be deployed early this summer. This new version will bring new features and capabilities, including authentication via encrypted username and password, a powerful database for handling thousands of VRVS users and more. The additional features are described in the following paragraphs.

\subsection{VRVS Current Product and Deployment Status}

\section{The VRVS Product Version 2.5}

During the last year, with the release of version 2.5, the VRVS software has been enhanced and consolidated to provide a solid base for distributed collaboration. The current features included multi-protocol, multi-OS support for all significant video enabled clients including: H.323, Mbone, QuickTime, MPEG2, Java Media Framework, and other clients. The current architecture makes VRVS a distributed, highly functional, and efficient softwareonly system for multipoint audio, video and web conferencing and collaboration over global IP networks. VRVS uses compact software running on inexpensive, standard networked computing server systems platforms to transparently interconnect standard clients over IP networks on a global scale.

Today, the system supports high-end, broadcast-quality interactivity, while enabling a wide variety of clients to participate in the same conference by running different standard protocols in different contexts with different bandwidth connection limitations.

VRVS is unique in providing a convenient, extensible, transparent, and client-agnostic multipoint architecture. It provides a wide range of functionality to let the user schedule, control and view a multipoint collaboration session, to choose among several client video formats, to tradeoff resolution and interactivity against bandwidth in bandwidth-limited situations, and to view, share, and manipulate documents across the world. Because of its peerto-peer network and modular architecture (as shown in Figure 1), VRVS can be adapted to a wide variety of different client application.

\footnotetext{
${ }^{2}$ See http://www.accessgrid.org
} 


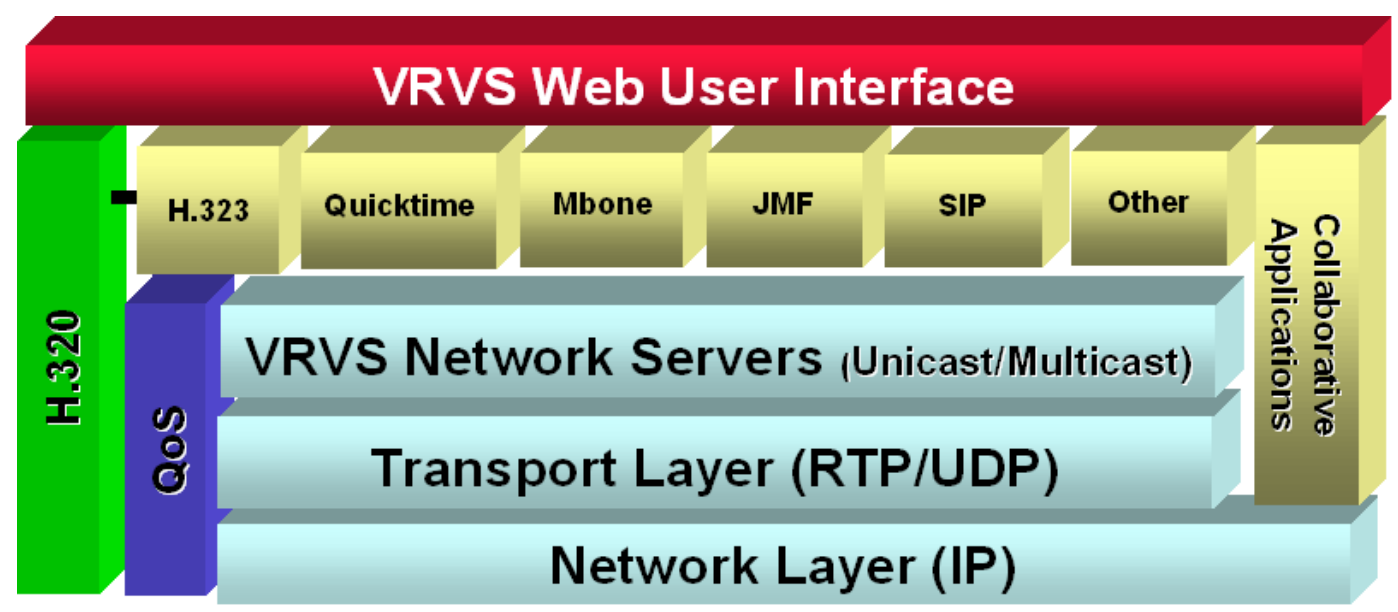

Figure 1: VRVS model Implementation

\section{Community of Users}

At the present time, more than 6,000 people in 60 countries have registered 10,500 machines in VRVS. Comparing these values with the figures 12 months ago (3,190 people and 5,190 machines), VRVS has increased by $100 \%$ its number of users and by 115\% the machines registered. Figure 2 shows the evolution of users and machines since January 2000. Figure 5 shows a typical meeting. VRVS started to grow within the High Energy Physics and Nuclear Physics communities, but due to its ease of use, flexibility of applications and devices, stability, support for the latest technologies and because of demonstrations during international events, today it encompasses many other communities. For example, VRVS is highly used in Astronomy, Fusion, Medicine, and other sectors of the Educational and Research communities worldwide.

The significant growth in the number of users during the last months means that people who regularly use the system are generally pleased with it, and those who try it for the first time find it very useful and well suited to their needs. The LISHEP-2002 conference $^{3}$ in Rio de Janeiro, Brazil, for example, generated much positive feedback on VRVS, and made the system known to many professors and students of diverse universities and research centers. Several remote presentations in real time showed them how straightforward it is to join a Virtual Room and interact with colleagues in other countries, and on one or more other continents.

Since the end of this conference the UERJ (Universidade do Estado do Rio de Janeiro) has initiated regular meetings among HEP groups at Fermilab and at universities in Brazil. For them, VRVS is an attractive tool because it is cheap, works fine with their "limited" networks in terms of bandwidth and QoS, and lets them organize their work without difficulty.

\footnotetext{
${ }^{3}$ See http://www.lishep.uerj.br
} 


\section{Machines and Users registered in VRVS}

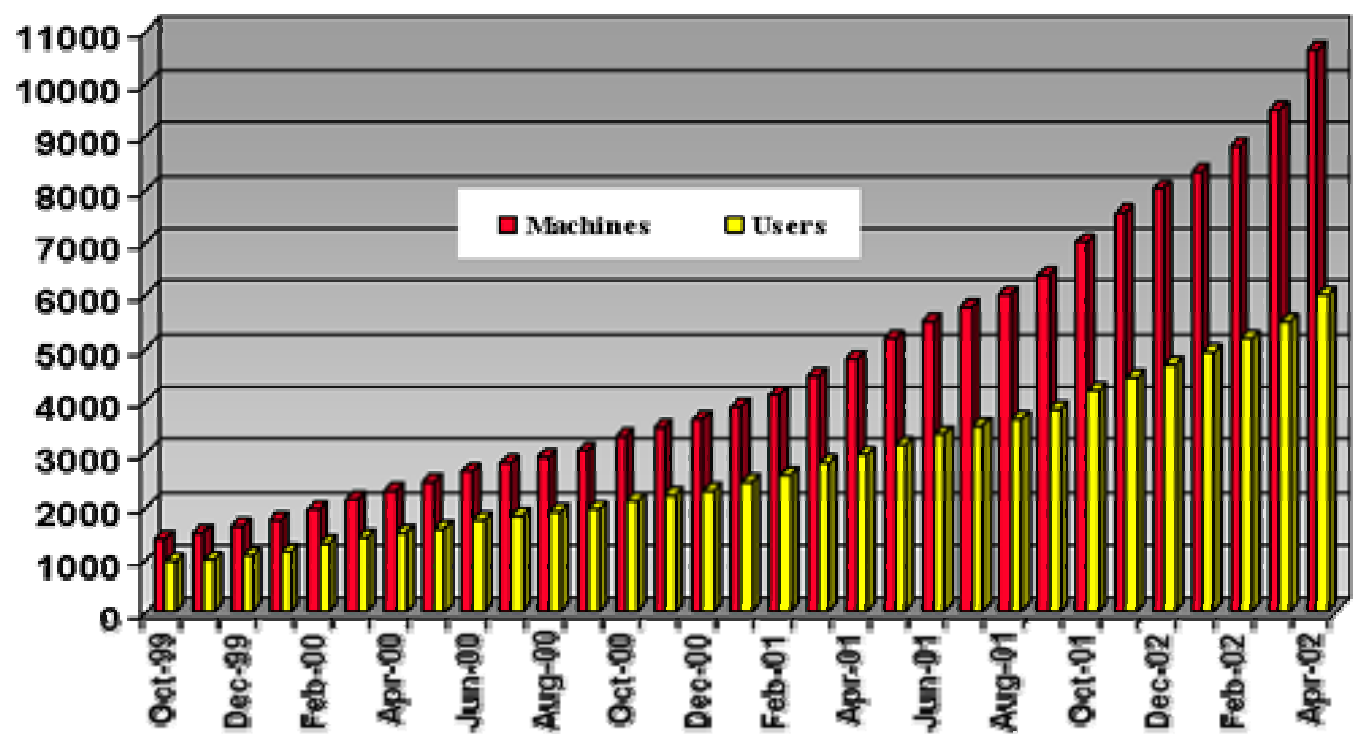

Figure 2: Registered Machines and Users in VRVS through April 2002

\section{Scheduled Conferences}

As a consequence of the increased number of users and interest in the system, the number of hours reserved to perform videoconferences and collaborative meetings has also increased at a remarkable rate. During the period May 2001 - April 2002 (both included), our users booked 10,288 hours of videoconferences.

This means a 109\% increase in relation to the previous 12 month period (May 2000 - April 2001), when they booked 4,929 hours.

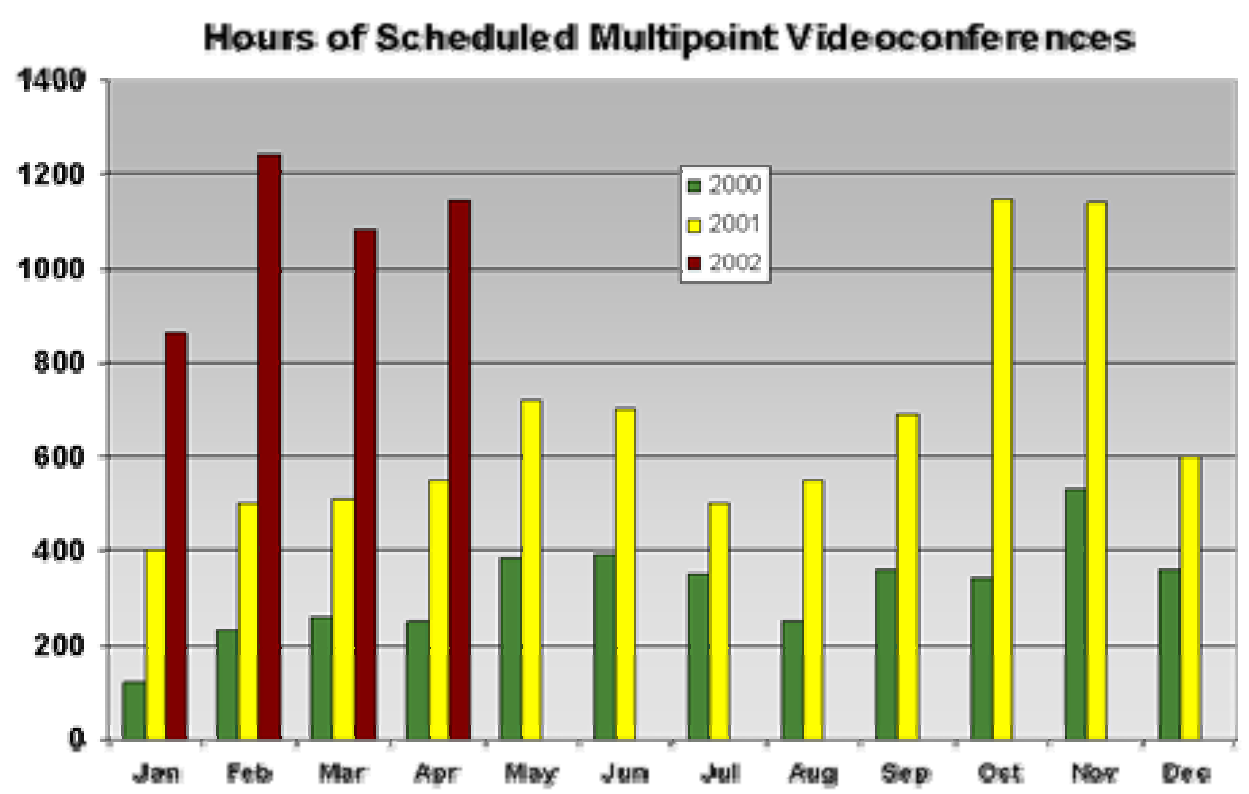

Figure 3: Number of Hours Booked for multipoint Videoconference session 
Last year the deployment of 5 new reflectors in the Internet2 backbone, and additional reflectors in Moscow, Romania, Portugal, and a second one in Israel, has made VRVS a solid and reliable infrastructure for remote collaboration within the United States and among North America, Europe, Middle East and Asia. During the last months, with the aim of improving IP communications and satisfying users' demands, VRVS is also expanding in Central and South America. In this context, we have installed two new reflectors in Rio de Janeiro, Brazil, and another one in Miami, Florida, at the AMPATH ${ }^{4}$ "Gateway to the Americas" Point of Presence. The AMPATH reflector is now the main entry point into the U.S. for VRVS traffic from Central and South America.

At the moment, the VRVS topology (Figure 4) is based on 42 reflectors (Linux PCs) located in 18 countries: the US (Argonne, BNL, Caltech (2), Fermilab, Florida (2), Internet2 (7), Jefferson Lab, SLAC), Brazil (3), Canada (2), Czech Republic, Spain (2), Finland, Israel (2), Italy, Japan, Poland, Portugal (2), Romania, Russia, Slovakia, Switzerland (2), Taiwan, U.K. (3), and Venezuela, routing the audio and video packets around the world. In the coming weeks, these values will continue to increase with the installation of new machines in Spain and the US.

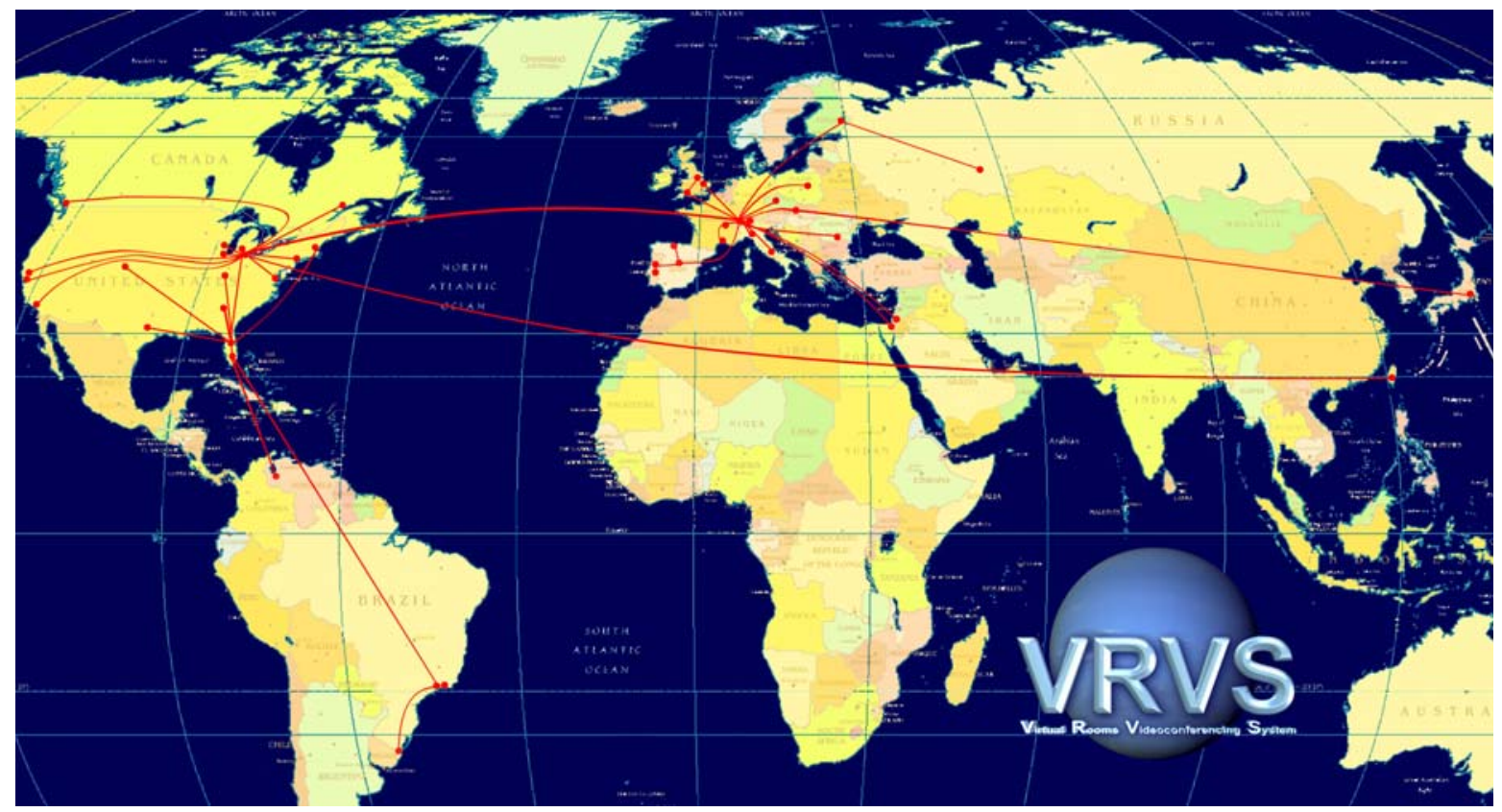

Figure4: Deployment of VRVS Reflector throughout the world

\section{Support for Multiple Autonomous Communities}

The VRVS system is currently used by several different Research communities represented by an experiment, project or a different research discipline. The communities that used intensively VRVS to perform their research are HENP (CMS, ATLAS, ALICE, LHC-B, CDF, DO, RHIC, etc), ESnet, Internet2, EFDA ${ }^{5}$, GLAST ${ }^{6}$ and LIGO$^{7}$. In addition to these general communities, inter-disciplines projects mainly Grid related projects are regular users of the VRVS system as well. Among this Grid projects we found PPDG ${ }^{8}$, GriPhyN ${ }^{9}$, DataGrid ${ }^{10}$, DataTag ${ }^{11}$.

As described in the following paragraphs, the VRVS architecture is being redesigned to allow the system to support several different autonomous communities, each of whom collaborate mainly within its own confines. In

\footnotetext{
${ }^{4}$ See http://ampath.fiu.edu

${ }^{5}$ See http://www.jet.efda.org

${ }^{6}$ See http://glast.gsfc.nasa.gov

${ }^{7}$ See http://www.ligo.caltech.edu

${ }^{8}$ See http://www.ppdg.net

${ }^{9}$ See http://www.griphyn.org

${ }^{10}$ See http://eu-datagrid.web.cern.ch/eu-datagrid

${ }^{11}$ See http://datatag.web.cern.ch/datatag
} 
general each community will be able to work without interacting with the other communities, thereby keeping the look and feel of the system relatively simple as overall use of VRVS grows.

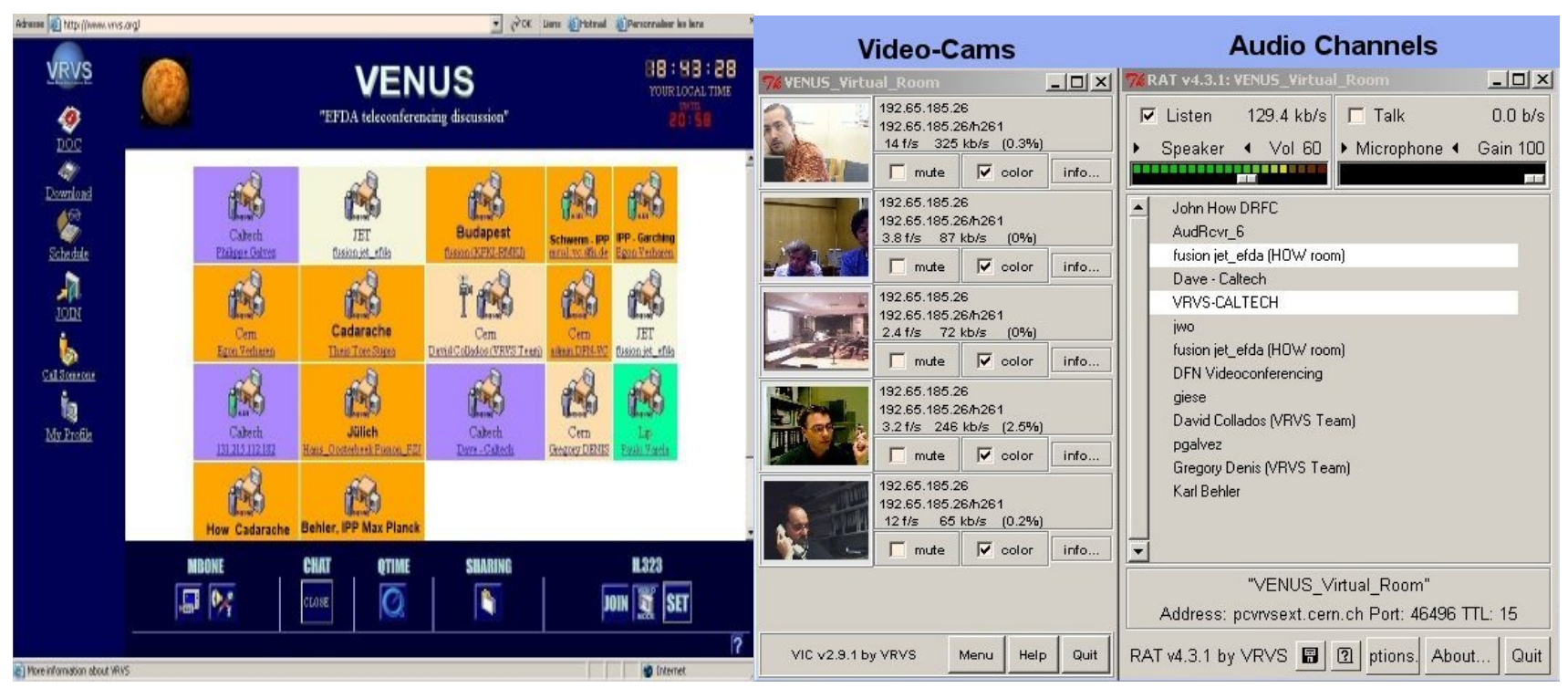

Figure 5: Typical VRVS Meeting

\subsection{VRVS Ongoing Development}

The VRVS system in production has been improved step by step over the years. The system has been under continuous development since 1997 when it first went into production, but because of its round-the-clock use, we have been careful to deploy the many new features, required to meet users' needs, in a series of controlled major software releases.

\section{New VRVS Server Architecture}

Because of the increasing use of VRVS, for several months now we have seen the saturation of the production server despite its stability and the improvements done. In order to cope with the increased success of our system we have decided to implement a server that is able to meet the current and future needs with the highest performance.

To do so we started a few months ago to re-architect VRVS. Up to now, 70\% of the development of our new VRVS server (Version 3.0) has been completed. The database design and setup, speed improvements by re-writing modules and scripts, security, booking, and many other improvements have been done. The connection process and some parts of the graphical user interface are still in development. We are confident of being able to fully release Version 3.0 in production during the Summer of 2002 (in August or September).

With performance in mind, MySQL ${ }^{12}$ server has been chosen to be our new database engine. We designed a new database in a flexible way to be able to face future needs easily. To seamlessly migrate to the new version, specific programs have been developed to convert the most important part of the current database to the new one. For example, some Virtual Rooms have already been booked far into 2003, and these bookings must be migrated systematically (more than 7000 entries).

In the new server version we have redesigned the web interface based on the requirements of our users and we made efforts to streamline the interface for efficiency and ease of use. The goal of the new graphical user interface is to deal with several communities, comprising hundreds of Virtual Rooms and tens to hundreds of thousands

${ }^{12}$ See http://www.mysql.org 
users, while providing more features and, at the same time, being very intuitive and easy to work with. The interface has been designed to be more adaptable: all the pages will be dynamically generated to fit automatically with the evolution of the system. One of the most difficult parts is to provide an intuitive and easy to use interface to find and join a meeting in a large number of parallel sessions.

Since we have users on 5 continents and many conferences span multiple time zones, an area of great concern to our users is the seamless and consistent scheduling of conferences across time zones.

The user has the ability to select his exact time zone and location via a graphical interface. The daylight saving summer time (DST) will be automatically managed for each country and region. In this way, the user will not have to take of it manually during the switching period ${ }^{13}$.

The new interface (Figure 6) gives the ability to the user to change dynamically the time zone and see the result instantaneously in all the pages (especially dates and times displayed). This will help users in coordinating conferences over multiple time zones. In the same way, the user can switch from one Virtual Room to another one in one click, and see immediately the result.

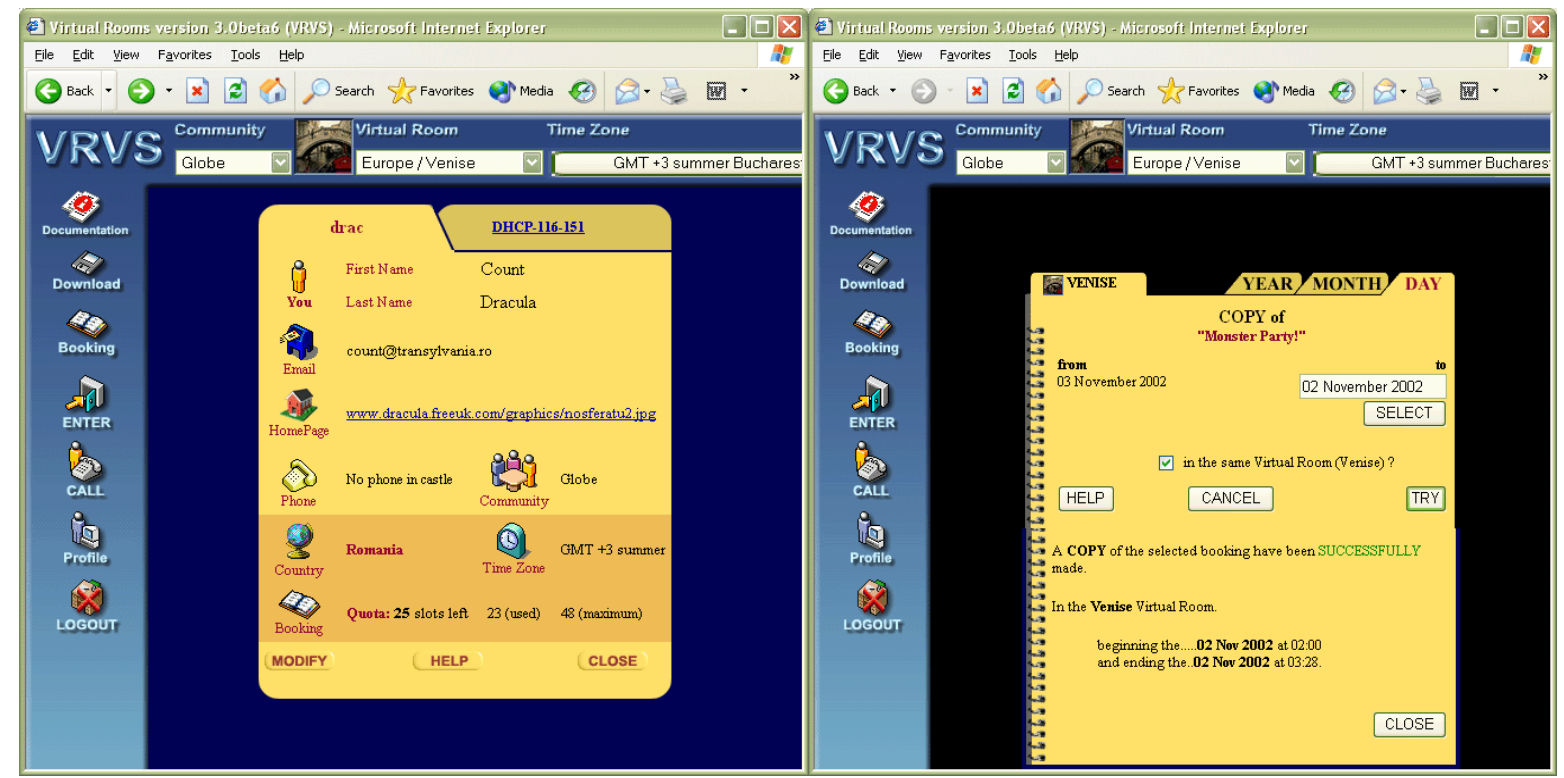

Figure 6: New interface for VRVS User Profile, Calendar with time zone, and meeting copy/paste

With the new database the booking system is more powerful and user-friendly. A user can now easily change his reservation's starting and ending times. We provide a fully new interface to allow the user to copy or to move his reservation to another date. The booking system transparently computes dates and times to be displayed in the selected time zone. In the booking form the user can also declare a mailing list to which information of a meeting's booking will be sent. In this way all participants of a meeting can be notified.

\section{Server Security and User Authentication}

Security is one of the main aspects we focused on when we developed the new version of the VRVS server. We used SSL encryption to protect the most sensitive part of our web interface (registration or authentication, for instance). A VRVS user will have to authenticate himself/herself to have access to our services (via the given user name and password). The authentication process is encrypted and checked directly with our main database.

In this way, we are sure of the user's identity wherever he is. Thus, we will be able to restrict more precisely the access to specific meetings, data, and we will allow users to use any machine (as opposed to the current system that has machines registered in the system).

\footnotetext{
${ }^{13}$ twice a year in the countries or states that support the DST
} 


\section{$\underline{\text { Resource Management }}$}

As the number of scheduled conferences increases, the demand for additional Virtual Rooms has also increased. Besides adding new rooms to the server, we have added ways to allocate rooms to groups or communities, and will add ways to limit the bandwidth conferences will use.

Our users have different bandwidth needs and capabilities. For this reason we put an option in the reservation system to allow the user to set minimum and maximum bandwidth limits that will be used by each participant during the future meeting.

The new version of VRVS handles several communities and sub-communities. It will be able to provide specific Virtual Rooms and features specifically adapted to the needs of each community. Users have the possibility to choose between the general Virtual Rooms (the ones that currently exist) and those specific to their community. A person attached to one community is able to enter his profile, including discipline-specific information related to his/her community. The system is flexible enough to handle any new community in an automatic way.

To improve the use of the Virtual Rooms we will add a quota management system. This system forces the user to book only the slots needed and to delete a reservation when the meeting has been canceled.

We have built a new machine detection process on the server which is more performant and works with the majority of web browsers and configurations. The user's machine no longer needs to be registered in the DNS (Domain Name Services). This improvement allow faster and more reliable discovery of the nearest reflector of a given machine. The new server architecture is able to manage machines behind a NAT (Network Address Translation) but some additional parts still needs to be implemented and tested in the reflector code to provide a complete solution.

\section{Improved Network Server (Reflector)}

To provide increased compatibility with as many video conferencing clients as possible, we have made many improvements to the reflectors. We have greatly improved the voice-activated video switching for use especially with the H.323 clients (which shows the current speaker in the one video display capable client).

We found that most of the H.323 commercial products are not 100\% H.323 compatible and therefore fail sometimes to communicate with each other. We managed to fix some of these broken standard H.323 pieces in our VRVS network servers to provide full interoperability between different non-standard H.323 clients when they are connected via VRVS.

We succeeded in bypassing the limitation of the QuickTime player by adding specific code in our last version of the reflector. Thus, the video displayed in that type of client switches automatically as it has been done for the H.323 clients. Moreover the QuickTime player is now able to play the audio from several participants at the same time.

\section{Improved Public Domain Tools}

From the start of VRVS we have supported the Mbone audio and video tools developed by UCL ${ }^{14}$. Since they are still heavily used by VRVS users we still support them and have modified them to be more compatible with VRVS. We have also enhanced these tools to deal with the latest devices, drivers and operating systems (especially for Linux and Windows).

And as application/desktop sharing adds much more utility to any cooperative system and as such is becoming more and more important to the VRVS users, we have enhanced AT\&T's VNC Java client ${ }^{15}$ to use the full color capabilities of the video display adapter.

\section{Integration with Access Grid}

${ }^{14}$ See http://www-mice.cs.ucl.ac.uk/multimedia/software

${ }^{15}$ See http://www.uk.research.att.com/vnc/ 
VRVS has developed the VRVS-AG Reflector and a specialized Web interface that enables end users to connect to any Access Grid (AG) session, in any of the AG "virtual venues" from anywhere worldwide. We call this the "Virtual Access Grid" (VAG). Users of the VAG have more flexibility, by being able to collaborate in the AG style, but without the usual AG requirements of a large set of room-based hardware, multicast and very high bandwidth network connectivity.

Because of the adaptability of the VRVS system software, the VRVS-AG Reflector and Web interface was completed in a period of a very few weeks.

The VRVS-AG Reflector provides transparent multicast/unicast switching to the users through VRVS web interface $^{16}$. End users can participate in any videoconferencing sessions in any AG Virtual Venues by using either Mbone (VIC/RAT) tools or H.323 clients. VRVS users send unicast video/audio streams to the corresponding AG Virtual Venue.

VRVS users can use the VRVS or AG or any other (openMash) Mbone tools to participate AG sessions through the VRVS web interface. RAT version 4 is required to participate in AG sessions. For H.323 participants, Access Grid nodes need to use a VIC version including a bug fix for the H.261 encoding protocol. We developed an audio transcoder that allow audio format used by the AccessGrid node, namely L16-16kHz to be converted to PCM (uLaw) at $8 \mathrm{kHz}$ which is the audio format supported by H.323 clients (following the H.323 I.T.U standard). Therefore, we have a full and transparent interoperability between H.323 and AccessGrid via VRVS.

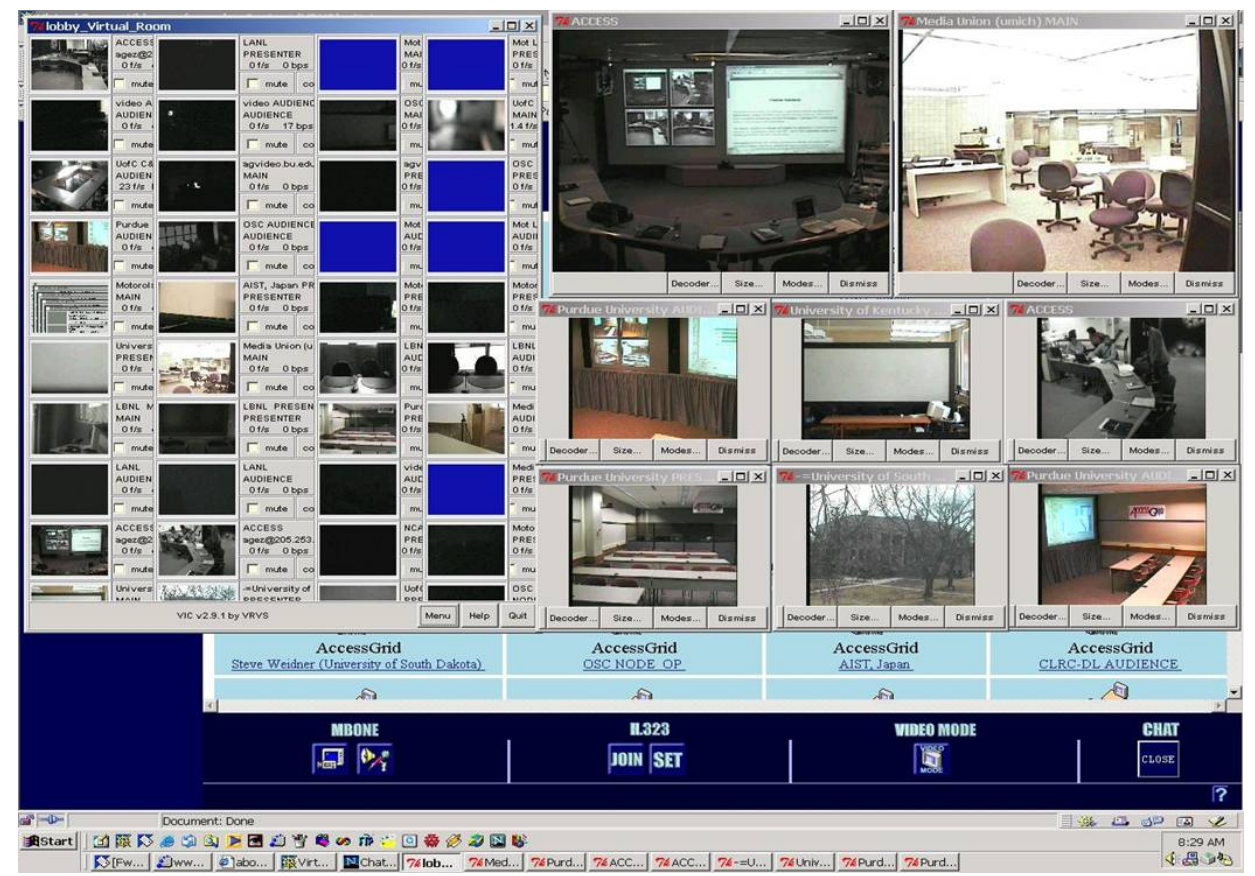

Figure 7: VRVS-AccessGrid Mbone Integration

In order to conserve network bandwidth and desktop CPU processing, we have set up the interface in such a way that a VRVS user is limited to only one video stream at a time ${ }^{17}$. With the Mbone tools that are part of the VAG, the user can receive all the active audio streams. A VRVS user also has the possibility to control which incoming video to receive. There are two video modes available now:

\footnotetext{
${ }^{16}$ See http://www.vrvs.com/accessgrid

${ }^{17}$ It is very easy to alter the VAG to allow the user select a subset of several live video streams in the AG venue. This change, planned for the near future, will be particularly effective for higher-end PCs such as Pentium 4's connected with switched Fast Ethernet.
} 
- Selected Participant Mode to select the specific video you want to watch from the participants list. This mode is useful for lectures and seminars.

- Browse Video Mode to watch each video site in turn. Video sites are selected in a "round-robin" queue that switches according to a preset timer.

Since the release of the VRVS integration with the Access Grid (VAG), many users have connected to Access Grid sessions through VRVS successfully, such as at the Supercomputing 2001 conference. It also has raised great interest among the in AG community (at the sites hosting AG nodes). VRVS has provided HOWTO documentation to the $\mathrm{AGDP}^{18}$ documentation Website, and tutorials and seminars to both the VRVS and AG communities.

Further developments on VAG include supporting more audio codec protocol bridging between VRVS unicast and AG multicast.

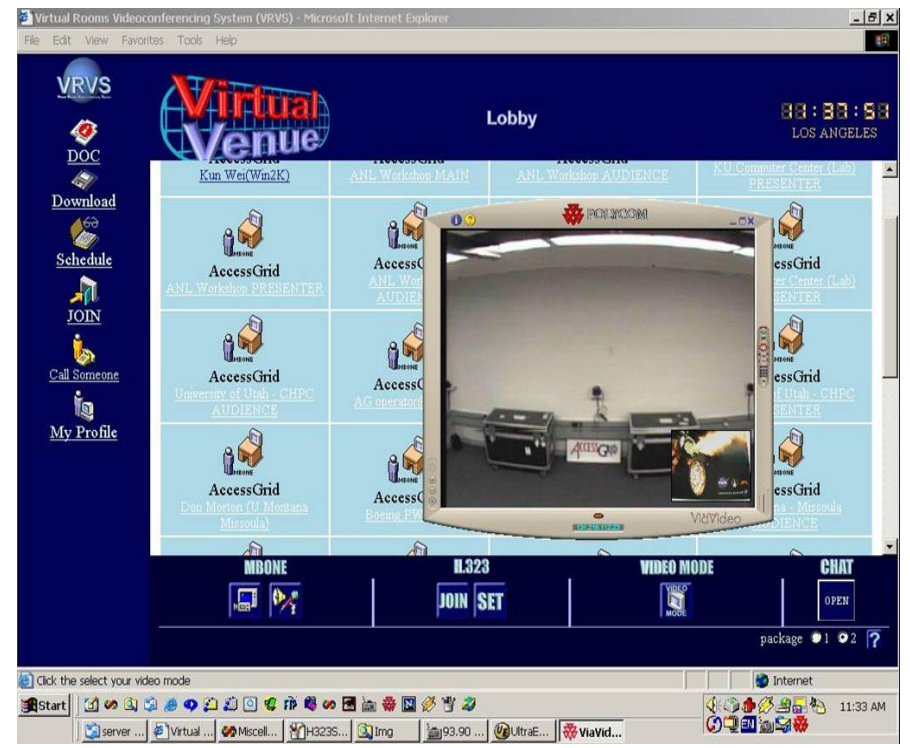

Figure 8: VRVS-AccessGrid H.323 Integration

\section{$\underline{2.4 \text { VRVS and Internet2 }}$}

Following the launch of the Internet2 project named the "Internet2 Commons" last year, a closer relationship between Internet2 and VRVS has been established. The Internet2 Commons project ${ }^{19}$ is a framework for collaboration throughout the research and education community that will encourage large scale deployment of tools for one-to-one, one-to-group, and group-to-group collaborations. These interactive communications include meetings, conferences, and activities related to teaching and learning. In this context, VRVS is a key element of the framework. It will provide scalability, reliability and usability for the Internet 2 community as well as provide gateways between different technology or areas (e.g. AccessGrid, Mbone, H.323) which is essential for this large and disparate users research community.

As part of the Internet2 Commons initiative, Internet2 and VRVS Caltech team deployed a series of VRVS servers, known as reflectors, over the Abilene backbone ${ }^{20}$ to both provide better performance for existing VRVS users and

\footnotetext{
${ }^{18}$ See http://www.accessgrid.org/agdp/howto/vrvs.html

${ }^{19}$ more information can be found at: http://commons.internet2.edu

${ }^{20}$ Abilene is an advanced backbone network that supports the development and deployment of new applications developed within the Internet2 community
} 
to facilitate access by new users. In addition to the two reflectors at the Internet2 Ann Arbor office and Indiana University, five additional reflectors have been installed and are available to the Internet2 Member Community. The location for these new reflectors are New York, Denver, Orlando, Texas and Atlanta.

Recently, the Internet2 management set up an independent task force to focus on scaling up the current existing videoconferencing effort (which includes VRVS) to a supported production service within the Internet2 community and international partners. This study will include a funding model and active participation of Internet2 members (more than 190 US Universities). The report from this task force is due by June 2002.

The VRVS team is also involved in the Internet2 Middleware Initiative (I2-VidMid). VidMid will initially focus on resource discovery and authentication for point-to-point and multi-point videoconferencing, and similar middleware requirements for video on demand, data collaboration, and voice over IP.

Because of the nature of VRVS, its capability for supporting real-time applications within large communities, and its ability to support both multicast and unicast technologies, other Internet2 Initiative and Working Groups expressed high interest in collaborating with VRVS. Such groups are for example, the Internet2 End-to-End Performance Initiative ${ }^{21}$ as well as the Internet2 QoS and Internet2 Multicast Working Groups.

\subsection{Next Generation Systems}

\section{$\underline{3.1 \text { Next Generation Systems }}$}

The VRVS system has now been running in production for five and half years, and it offers to the HENP community a working and reliable tool to collaborate within groups and among physicists dispersed world-wide. The next generation of collaborative systems (based on current VRVS development) will evolve to be more scalable and reliable, to integrate new emerging standards, to support new products to provide very high-end quality, and to provide state-of-the-art efficiency in terms of quality per unit bandwidth. More specifically, we intend to enhance the system by working on a number of areas:

- Adaptation to Internet emerging standards such as IPv6 ${ }^{22}, \mathrm{SIP}^{23}$, and IEEE 802.11(a\&b) ${ }^{24}$.

- Integration of new hardware compression devices for high end quality interaction (e.g. MPEG2, MPEG4, HDTV $^{25}$ )

- Enhancement of the system to handle increasing deployment of a scalable network security infrastructure. This deployment includes the installation of firewalls and secure methods for traversing them where needed, $\mathrm{NAT}^{26}$ handling, and other secure mechanisms incompatible with open communication and collaboration between geographically separated sites.

- Development of advanced Monitoring and Tracking tools to handle the exponential growth of VRVS userto-user ad hoc conferences as well as ongoing scheduled multisite conferences.

- Development of a pure peer-to-peer reflector network in order to provide high reliability communication network as well as high scalability to handle thousands of parallel sessions (and a correspondingly large number of video and audio streams).

- Development of new features and functionality as continuously requested by the VRVS community to fit their particular needs.

For several years now, we have been witnessing the explosive growth of Internet usage. To meet this exponential

\footnotetext{
${ }^{21}$ See http://www.internet2.edu/e2e

22 Internet Protocol Version 6. See http://www.ipv6.org

${ }^{23}$ Session Initiation Protocol. See http://www.ietf.org/html.charters/sip-charter.html

24 The IEEE wireless LAN standards

${ }^{25}$ High Definition Television

${ }^{26}$ Network Address Translation
} 
growth the industry body standards have, in collaboration with the Internet Engineering Task Force, developed new standards to scale and to be able to manage this technological revolution. Among such standards are IPV6 (vital for adequate IP address space for fixed and mobile devices), SIP, and 802.11(a\&b). These standards are now mature enough to be widely deployed within large network backbones, campuses, etc. VRVS next-generation systems will have to integrate these new standards within its architecture and modify code as necessary. The new system will have to be fully compliant with these standards and be ready soon for wide deployment.

In order to provide high-end interactivity, we have to continuously watch the video market for new hardware/software devices able to provide very high quality at reasonable cost, and integrate them into the VRVS architecture. We have already developed MPEG2 multipoint capability within VRVS using the Minerva Codec ${ }^{27}$. Driven by users' demand, we will support other MPEG2 manufacturers and/or find affordable codec solutions to be used by a larger community. Recently, new types of compression standards, able to provide high to very high quality videoconference with optimum interactivity, are becoming available for a larger audience (MPEG4 and to some extent HDTV). New developments to integrate these technologies is planned to be included in the next (Version 4.0) VRVS release.

Over the last few months there has been an increased tendency for universities and laboratories to consolidate their Internet security infrastructure. An increasing number of sites have installed firewalls (which prevent unauthorized IP packets to reach any host in the protected site) and/or NATs (which hide the original IP address to the rest of the world and use an internal non-routable IP address). The VRVS next-generation system will have to adapt to these new situations by accommodating these strong security requirements while overcoming the hindrances that they could present to open communication and collaboration between institutes. The solution and implementation must be light enough so no significant load or complexity would be added to the work of end-users or network administrators.

Today, with more than 1,100 hours of multipoint sessions per month and the exponential growth forecast for 20023 , we need advanced tools that will allow the VRVS administrators to monitor and check in real-time all of the ongoing conferences (from 10 to several thousand). The system should be able to track the past and current connections per user and per Virtual Room meeting and to provide clear statistics on various aspects of VRVS resource usage via an intuitive interface. For example, we should be able to request current and historical information for any particular VRVS user in detail, such as the last time he connected to the system, how many hours per week/month/year he used the system, which tools he used, and from which machine/location he connected to the system (especially in the case of mobile users).

Currently, all the VRVS reflectors are statically configured and managed via the web server. In order to contend with the foreseen increase in VRVS reflectors at new locations, we will need to redesign the way the reflectors interact with each other and with the web server. This new design, based on a pure peer-to-peer architecture, will facilitate the monitoring functions as well as providing a high degree of reliability and scalability. Each reflector will be independent, self-managed by internal monitoring agents, and will interact only with the peer reflector nodes. This schema will provide full redundancy, automatic rerouting of packet streams in case one reflector fails, automatic bandwidth and QoS monitoring (latency, packet loss, jitter) between reflectors to assure the best possible connectivity for real time communication, etc.

In the meantime, we continue to gather useful feedback and specific requests from the VRVS user community on a continuous basis. There are obviously constant demands for additional features and functionality to fit their needs. Our objective is to prioritize these requests and implement them in case they are technologically feasible and fit in the VRVS framework development plan.

\footnotetext{
${ }^{27}$ See http://www.minervanetworks.com
} 